\title{
An unusual high-ionization nebula in NGC 55
}

\author{
R. Tüllmann ${ }^{1}$ and M. R. Rosa ${ }^{2, \star \star}$ \\ 1 Astronomisches Institut, Ruhr-Universität Bochum, Universitätsstr. 150, 44780 Bochum, Germany \\ 2 Space Telescope European Coordinating Facility, c/o European Southern Observatory, Karl-Schwarzschild-Str. 2, \\ 85748 Garching, Germany
}

Received 26 August 2003 / Accepted 1 December 2003

\begin{abstract}
We report on the detection of a previously unknown extended low-density nebula of very high temperature and excitation, TR 001507.7-391206, located above the midplane of NGC 55. The nebular emission line spectrum, in which He II is present, is consistent with photoionization by about 3 very hot massive O3 or WR-type stars. There are no indications for shock ionization. The faint blue optical continuum and XMM-Newton EPIC-pn and OM-data also support our assumption that this nebula is not a supernova remnant. Galactic nebulae harboring massive Wolf-Rayet (WR) stars may be appropriate examples, in particular the highly ionized nebula G2.4+1.4 around WR 102 (aka Sk 4 or LSS 4368). The relatively large diameter of the inhomogeneously expanding nebula in NGC 55 of about 58 pc, compared to 11 pc of G2.4+1.4, would also be consistent with the number of ionizing stars.
\end{abstract}

Key words. stars: Wolf-Rayet - ISM: abundances - ISM: individual: TR 001507.7-391206 - galaxies: individual: NGC 55 X-rays: ISM

\section{Introduction}

During a study on the Wolf-Rayet (WR) star content of NGC 55, a SBm-type galaxy of the Sculptor-group (e.g., Graham \& Lawrie 1982; Ferguson et al. 1996; Otte \& Dettmar 1999; Tüllmann et al. 2003) with EFOSC1 at the ESO $3.6 \mathrm{~m}$ telescope in 1990, narrow-band images in $\mathrm{H} \alpha$ and [O III] $\lambda 5007$ revealed a faint, round, featureless nebular object. Contrary to the classical giant H II-regions nearby in the body of NGC 55, it did not show any central peaking of the nebular emission, nor did any stellar-like central source seem to be present in medium-band continuum images. One of the longslit spectra taken for the stellar cores of the giant H II-regions was placed across the nebula to yield a first test spectrum. As exposed for $1200 \mathrm{~s}$, the observed spectrum was very weak, but showed surprisingly strong [O III]-lines. Even [O III] $\lambda 4363$ was measurable, and yielded a rough estimate of $20000 \mathrm{~K}$ for the electron temperature. This is very high in comparison to values typical for the giant H II-regions in NGC 55 of about $11500 \mathrm{~K}$ (Tüllmann et al. 2003).

Send offprint requests to: $\mathrm{R}$. Tüllmann,

e-mail: tullmann@astro.rub.de

* Based on observations collected at the European Southern Observatory, La Silla \& Cerro Paranal (Chile); Proposal No.: 64.N0399(A,B), 66.B-0551(A). Based upon data obtained from the ESA XMM-Newton data archive.

$\star \star$ Affiliated with the Space Telescope Division of the European Space Agency, ESTEC, Noordwijk, The Netherlands.
The object certainly deserved further study, but it was only with more sensitive equipment that deeper spectra and images could be obtained in reasonable exposure times. A decade after discovery we now can benefit from deeper optical spectroscopy, images from the VLT, and UV/X-ray imaging from the XMM-Newton satellite.

In the following we present observational results from these multi-wavelength data and support the analysis with a detailed modeling of the nebular emission line spectrum in order to identify the ionization mechanism and to investigate the nature of the central ionizing source.

\section{The data}

\subsection{Optical spectroscopy and imaging}

The nebula, named TR 001507.7-391206 from now on, was spectroscopically observed in two different observing runs in June 1990 and October 2000 with ESO's Faint Object and Spectrograph Cameras EFOSC1 and EFOSC2, attached in both runs to the $3.6 \mathrm{~m}$ telescope located at La Silla observatory. For both spectroscopy runs we selected a rather wide slit of $2{ }^{\prime \prime} 0$ in order to gain maximum flux from the extended target while still maintaining a reasonable spectral resolution just high enough to make use of the density sensitive [S II] doublet at $6724 \AA$. Spectra collected with EFOSC1 in 1990 have a spatial resolution of $0.68^{\prime \prime} \mathrm{pix}^{-1}$, the value of those obtained with EFOSC2 in 2000 amounts to $0.157^{\prime \prime} \mathrm{pix}^{-1}$. 


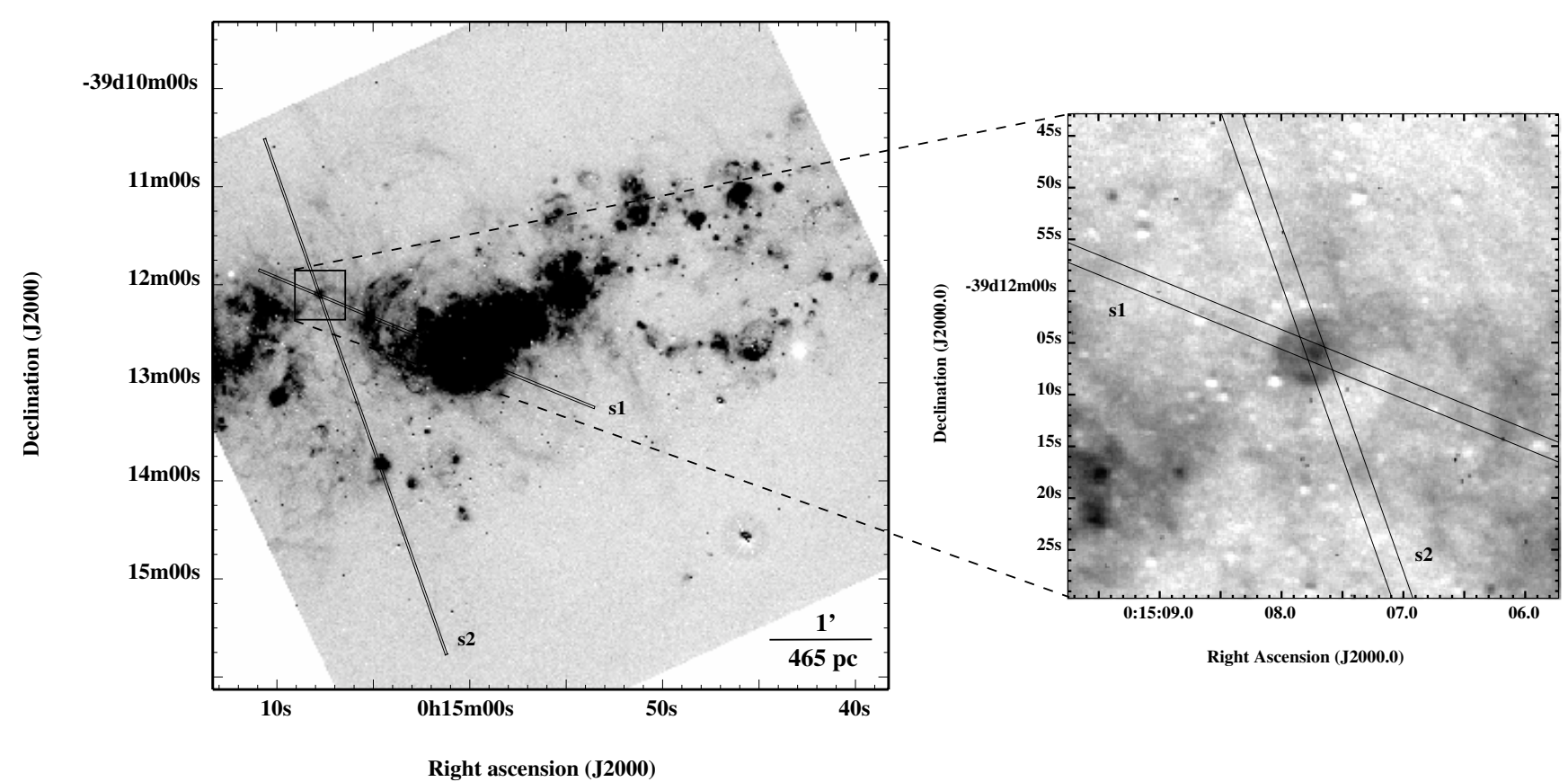

Fig. 1. This VLT H $\alpha$-image of the central part of NGC 55 was taken from Tüllmann et al. (2003). Slit positions $s 1$ (EFOSC1) and $s 2$ (EFOSC2) are scaled to match the chosen slit width of $2^{\prime \prime}$ and the individual slit lengths of both instruments. The magnified image to the right shows the position where both slits cut through the nebula.

Table 1. Journal of observations.

\begin{tabular}{lllll}
\hline \hline Telescope & Instrument & Date & $\begin{array}{l}\text { Spectral } \\
\text { region }\end{array}$ & $\begin{array}{l}\text { Duration } \\
{[\mathrm{ks}]}\end{array}$ \\
\hline $3.6 \mathrm{~m}$ & EFOSC1 & $06|20| 90$ & {$[\mathrm{O} \mathrm{III}], \mathrm{V}$} & $0.6,0.6$ \\
& & & $3650-10^{4} \AA$ & 1.2 \\
& EFOSC2 & $10|29| 00$ & $3500-10^{4} \AA$ & $1.2-3.6$ \\
VLT & FORS1 & $09|12| 99$ & $\mathrm{H} \alpha, R$ & $0.6,0.12$ \\
XMM & pn & $11|15| 01$ & $0.3-12 \mathrm{keV}$ & 28.3 \\
& OM & $11|15| 01$ & $\mathrm{U}$ & 0.8 \\
& & & UVW1 & 1.06 \\
& & & UVW2 & 2.3 \\
\hline
\end{tabular}

During the run in 1990 a number of direct images were obtained with EFOSC1 through narrow-band filters centered on [O III] $\lambda 5007,[\mathrm{O} \mathrm{II}] \lambda 3727, \mathrm{H} \alpha, \mathrm{H} \beta$, and [S II] 26727 , as well as continuum frames in medium-band Strömgren $b, y$, and broadband $R$. These images initially led to the discovery of the object but were of mediocre quality (focus and seeing) to yield any further insight.

In addition, however, we can make use of deeper broadband $R$ and narrow-band $\mathrm{H} \alpha$ VLT-images with much better spatial resolution obtained during an independent study (Tüllmann et al. 2003), covering the same region of the sky. The above reference also provides information on data reduction techniques for spectroscopy and photometry which are widely followed here as well. A journal of observations is presented in Table 1.

In Fig. 1 the VLT H $\alpha$-image is shown together with detailed positions and dimensions of the two slits $s 1$ and $s 2$. The magnified image to the right clearly reveals some substructure and indicates which parts of the nebula are covered by the longslits.

\subsection{XMM-Newton-data}

We also analyzed XMM-Newton archive data (Obs.-ID.: 0028740101, Rev.-No.: 0354) of NGC55 using primarily EPIC-pn (Strüder et al. (2001) and OM-data (Mason et al. 2001). Data reduction was done with standard tasks provided by SAS v.5.4.1.

After first inspection of the pn data, a light curve was created to check for flaring events and to select the good time interval (GTI). As no background flaring was found to be present, the full exposure time was usable (Table 1). The eventlist was filtered selecting PATTERN $\leq 12$ and $\mathrm{FLAG}=0$ and cleaned images were produced in the three energy bands $0.3-12 \mathrm{keV}$ (total), 0.3-2.0 keV (soft) and 2.0-12.0 keV (hard).

OM-data for the UV-filters U, UVW1, and UVW2 were pipeline processed and calibrated using the SAS-task OMICHAIN. The PSF ( FWHM) of all filters is $\leq 2$ '. 3 . Integration times for the corresponding filters are listed in Table 1. Parasitic light (e.g., visualized by straylight ellipses, rings, ghosts, etc.) at the position of the nebula turned out to be negligible.

Due to low count rates, no X-ray spectra could be extracted.

\section{Results and discussion}

If projection effects along the line of sight are irrelevant, TR 001507.7-391206 is located on top of an extended Vshaped column of gas and dust (seen upside down), that is protruding off the disk plane of NGC 55. This is shown very nicely in Fig. 1, where the gaseous structure is aligned with slit position $s 2$. 

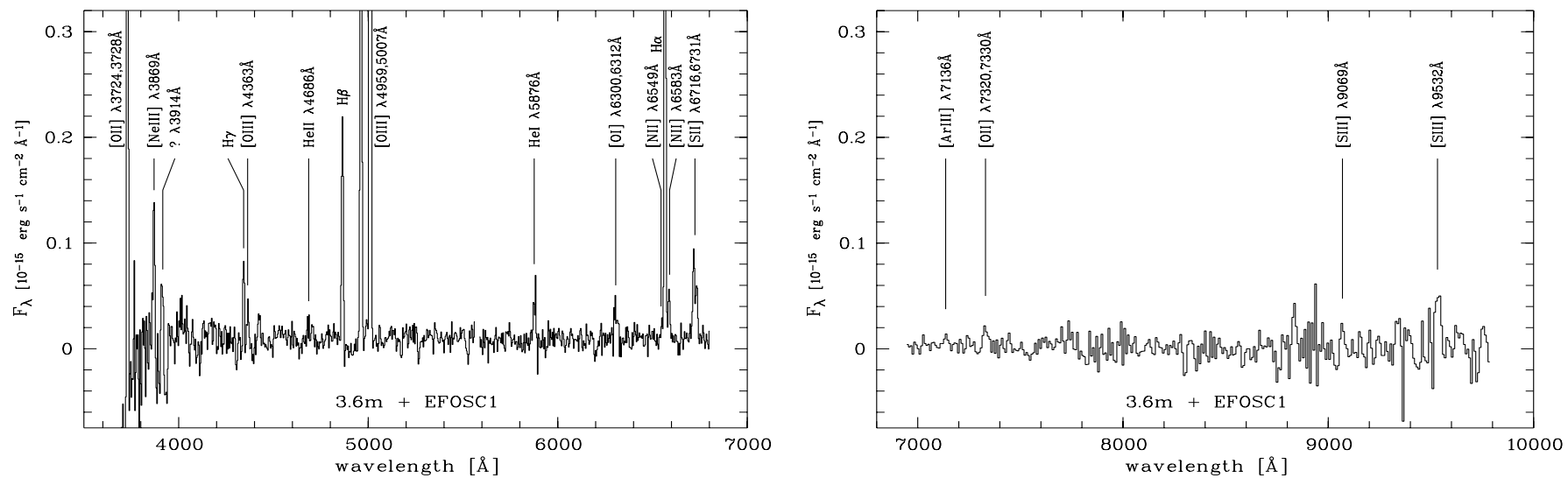

Fig. 2. Fully-reduced 1D-spectra of TR 001507.7-391206 obtained with EFOSC1 during the first spectroscopy run in June 1990 (slit $s$ ). The spectra are the sum of the emitted flux from the central part and the nebula. Most of the He II $\lambda 4686$ line emission detected at slit position $s 1$ is concentrated in the western and the central part of this object.
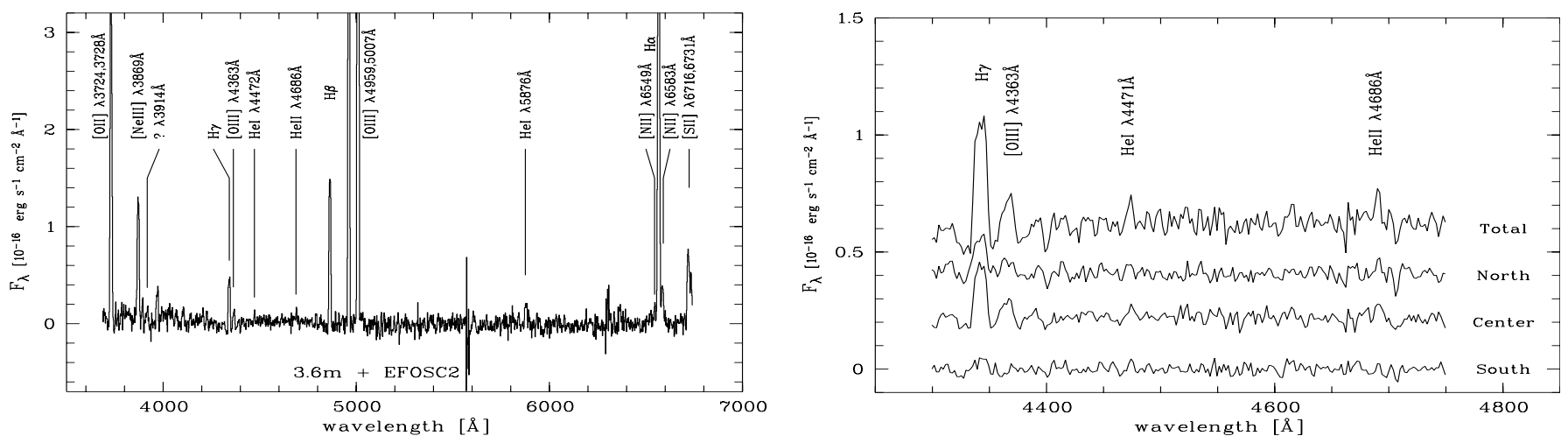

Fig. 3. This integrated spectrum of slit $s 2$ has been obtained with EFOSC2 during the second spectroscopy run in October 2000. Only in the center of the nebula very weak stellar continuum emission is detectable. Spectral plots, emphasizing He II $\lambda 4686$ and [O III] $\lambda 4363$ line emission, are displayed with reasonable offsets in the right panel and cut through different parts of the nebula (cf. Fig. 1).

A spectrum of the nebula covering the wavelength range from $3500 \AA-10000 \AA$ is presented for slit $s 1$ in Fig. 2. Since the airglow was much stronger during the EFOSC2 run, the spectrum for slit location $s 2$ is displayed only in the range of $3500 \AA-7000 \AA$ (Fig. 3, left panel).

In Fig. 3 (right panel) we also show spectra from slit position $s 2$ sampling different sections of the slit. These plots reveal that the total integrated emission of He II $\lambda 4686$ for slit $s 2$ is weak, but significant. Apparently, this emission is concentrated in the northern and central parts of the nebula, whereas there is no clear detection for the southern part. The co-added data for slit $s 1$ are of insufficient $\mathrm{S} / \mathrm{N}$ to investigate spatial variations of the faint He II $\lambda 4686$ line emission.

Although this emission line appears to be relatively broad at slit position $s 2$, it is completely unresolved, as the $F W H M$ is only half of that of $\mathrm{H} \gamma$ or [O III] $\lambda 4363$. Therefore, line broadening effects (e.g., due to stellar winds) are not supported by the data presented here. Since the He II $\lambda 4686$ emission is spatially extended, and the stellar continuum is extremely weak, it is safe to assume that all of the emission is nebular in origin (see also flux estimates below).

The spectrum of the red wavelength region obtained at slit position $s 1$ does not show stellar continuum emission at all. However, there is very faint continuum detectable within the central $15 \mathrm{pc}$ at slit position $s 2$ throughout the whole covered wavelength region. In addition, continuum is most likely present in the $V$-band image and, to a significantly lower extent, also on the $R$-band frame (cf. Fig. 4). Among the data presented here, this emission appears to be strongest in the UV broadband images obtained with the OM-telescope of XMMNewton (Fig. 5). The presence of a weak continuum, slightly stronger in the blue, is confirmed by the spectra shown in Fig. 2.

Although the spectrum of the potential central ionizing source is too weak to be extracted, we can constrain its nature indirectly by analyzing the ionization mechanism and measuring ionic and elemental abundances of the surrounding nebula.

Observed and dereddened emission line fluxes are listed in Table 2 together with predictions of the nebular abundance tool (NAT, see text below). Because of the high (negative) galactic latitude of NGC 55 almost all of the reddening is due to dust located in that galaxy. In the absence of a dedicated reddening law valid for NGC 55 we used the standard galactic law for dereddening. Even if we had used the optical part of the SMC-law the reported line ratios would not have changed significantly for the present purpose. 


\section{6m + EFOSC1}
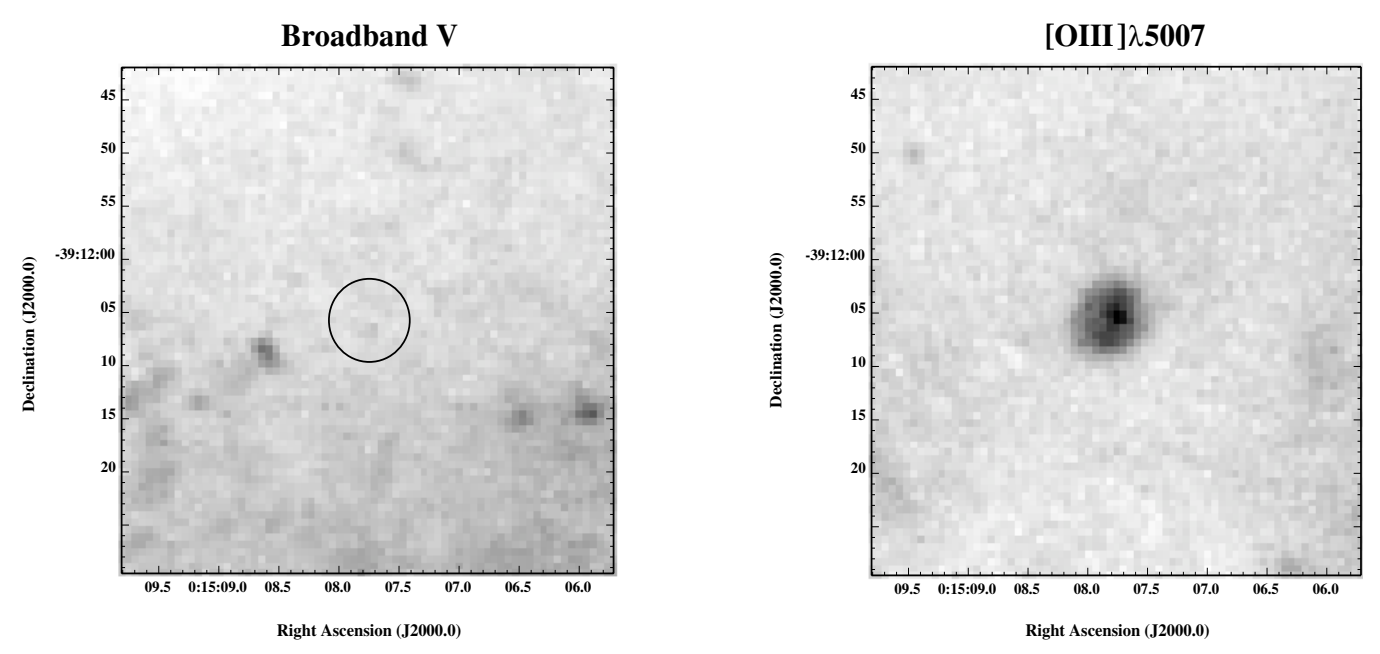

VLT + FORS1
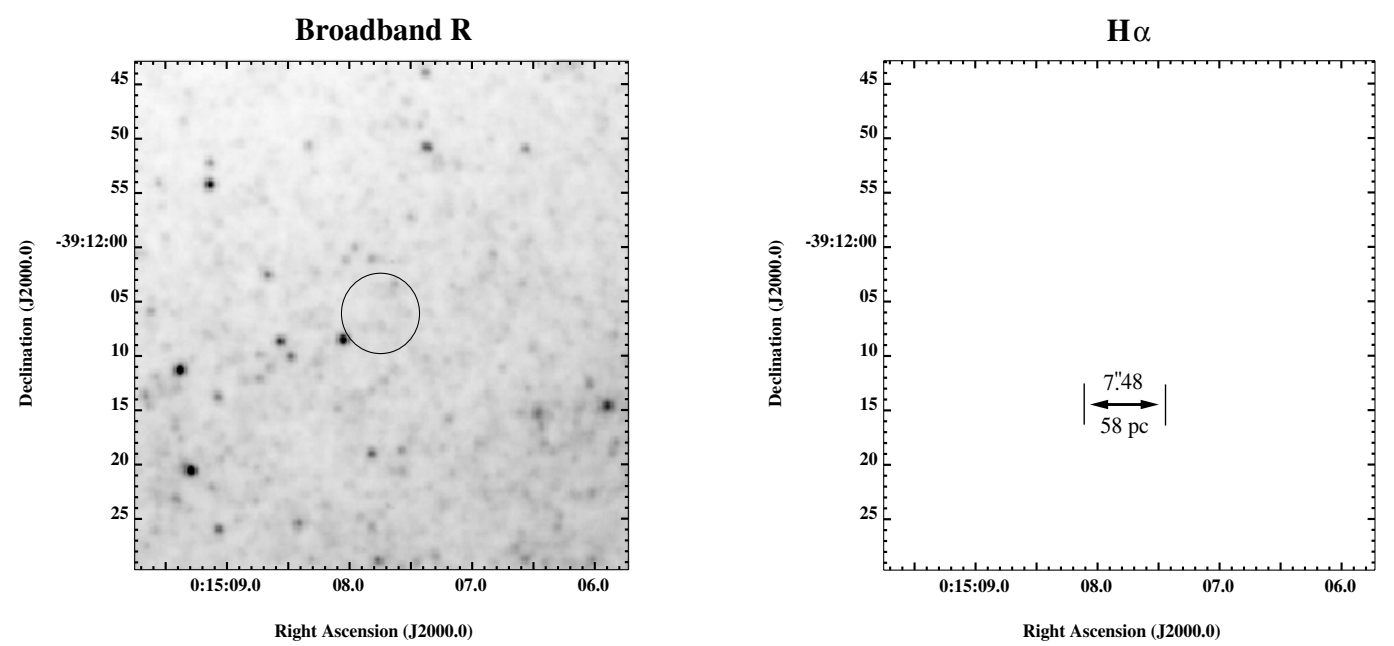

Fig. 4. Broad- and narrow-band filter images of TR 001507.7-391206 obtained with different ESO instruments. Due to better spatial resolution of the FORS1 instrument, the VLT H $\alpha$-image reveals a central dense core and a fragmented outer gas shell. There seems to be very faint continuum emission in the $R$-band filter image, which appears to be slightly stronger in the $V$-band. For $V$-and $R$-band photometry the location of the object is marked by a circle. The diameter of the nebula has been determined to be 7 '. 5 which corresponds to $58 \mathrm{pc}$.

\subsection{Gas phase abundances}

The direct detection of [O III] $\lambda 4363$ enables us to obtain a reliable estimate of the gas temperature and, together with constraints on the density, ionic abundances of the gas. Abundances are determined with NAT, a code based on the 5level-atom program of De Robertis et al. (1987) (see Tüllmann et al. 2003 for details). If only a single transition of an element could be observed (e.g., [Ne III] or [Ar III]), NAT adopts this value to calculate fractional ionizations and element abundances. However, since only photon energies $<54 \mathrm{eV}$ are considered, no ionic abundances are calculated for He II by this tool.

A final conversion from ionic to element abundances is achieved by applying the ICF scheme of Mathis \& Rosa (1991) which allows us to use parameterized results from photoionization models with a wide variety of stellar atmosphere models. Element abundances determined this way are given in Table 3.
For convenience, we consider the metallicity $Z$ to be equal to $\log (\mathrm{O} / \mathrm{H})$, since oxygen is the most abundant and efficient coolant.

In relation to the solar values (Christensen-Dalsgaard 1998; Grevesse \& Sauval 1998), $\left[\mathrm{He}^{+} / \mathrm{H}^{+}\right]$appears to be overabundant by about a factor of 2.5 at slit position $s 1$ and by about a factor of 1.5 at position $s 2$. The total atomic $\mathrm{He} / \mathrm{H}$ ratio will be slightly higher if the ionization of $\mathrm{He}$ II is accounted for. $[\mathrm{Ne} / \mathrm{O}]$ at position $s 2$ appears to be overabundant by about the same value of 1.5. However, Ne-abundances are based on only a single $\mathrm{Ne}^{++}$transition at $3869 \AA$ and are therefore less certain. At both slit positions the $[\mathrm{S} / \mathrm{O}]$-ratio also turns out to be enhanced by an average factor of 1.3 .

The ionization of the nebula is obviously rather high. At slit position $s 1(s 2)$ only $21 \%(34 \%)$ of oxygen is expected to be present as $\mathrm{O}^{+}$and $79 \%(66 \%)$ as $\mathrm{O}^{++}$. Therefore, it is remarkable that the oxygen abundance of TR 001507.7-391206 

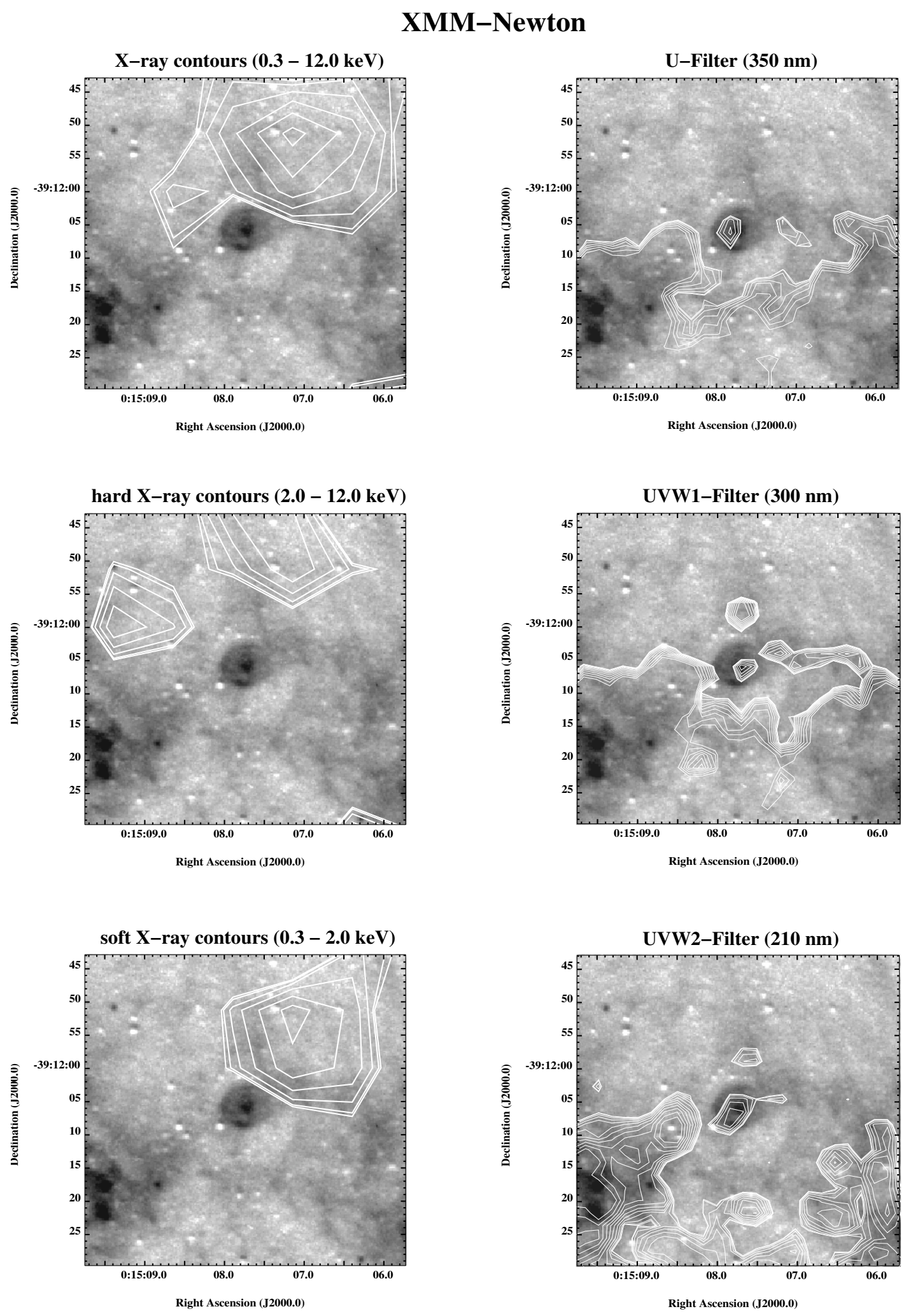

Fig. 5. XMM-Newton view of the same region. X-ray contours (EPIC-pn data, left panel) for different energies are overplotted on the VLT H $\alpha$ image. In addition, UV broadband contours from the optical monitor (OM, right panel) unambiguously reveal continuum emission indicating most likely the presence of a hot stellar source in the center of the nebula.

is within 0.1 dex identical to that found for the extraplanar H II-regions (EHRs) in the disk-halo interface of NGC 55 (Tüllmann et al. 2003). Similar to the EHRs, the nebula is also located above the central star forming complex, which would imply that the EHRs and the nebula have been formed from the same gas that has been pushed into the halo by the combined effects of stellar winds and supernova explosions during an earlier epoch of star formation in the galactic disk of NGC 55. 
Table 2. Dereddened emission line fluxes for both slit positions listed together with corresponding NAT predictions. Since the spectral resolution is too low to resolve [O I] $\lambda 6300$ and [S III] $\lambda 6311$, the sum of both lines is given instead. The same holds for the oxygen-doublets at $3727 \AA$ and $7325 \AA$.

\begin{tabular}{|c|c|c|c|c|}
\hline $\begin{array}{l}\text { Line } \\
\mathrm{H} \beta=100\end{array}$ & slit 1 & NAT & slit 2 & NAT \\
\hline$\Sigma$ [O II] $\lambda 3727$ & 315 & 315 & 499 & 500 \\
\hline [Ne III] $\lambda 3869$ & 83.9 & 83.9 & 156 & 156 \\
\hline $\mathrm{H} \gamma$ & 34.3 & 47.5 & 46.9 & 47.5 \\
\hline [O III] $\lambda 4363$ & 23.3 & 23.2 & 19.6 & 19.3 \\
\hline Не г $\lambda 4472$ & - & 10.3 & 5.62 & 6.49 \\
\hline He II $\lambda 4686$ & 4.88 & - & 2.60 & - \\
\hline $\mathrm{H} \beta$ & 100 & 100 & 100 & 100 \\
\hline [О Ііл] $\lambda 4959$ & 276 & 279 & 227 & 233 \\
\hline [O III] $\lambda 5007$ & 814 & 805 & 686 & 670 \\
\hline [Ar III] $\lambda 5193$ & - & 0.24 & 2.42 & - \\
\hline$[\mathrm{Cl}$ III $] \lambda 5518$ & 9.54 & 9.54 & 0.88 & 0.88 \\
\hline Не I $\lambda 5876$ & 27.4 & 27.4 & 19.5 & 17.2 \\
\hline $\left.\begin{array}{l}{[\mathrm{O} \text { I }] \lambda 6300} \\
{\left[\mathrm{~S}_{\mathrm{III}}\right] \lambda 6311}\end{array}\right\}$ & 36.1 & 4.42 & - & - \\
\hline$[\mathrm{N}$ II] $\lambda 6548$ & 5.05 & 5.83 & 3.81 & 6.43 \\
\hline $\mathrm{H} \alpha$ & 274 & 274 & 274 & 274 \\
\hline [N II] $\lambda 6583$ & 19.5 & 17.2 & 26.7 & 18.9 \\
\hline [S II] $\lambda 6716$ & 40.5 & 36.0 & 54.2 & 48.5 \\
\hline$[\mathrm{S}$ II] $\lambda 6731$ & 22.1 & 25.3 & 30.0 & 34.1 \\
\hline [Ar III] $\lambda 7136$ & 11.3 & 11.3 & - & - \\
\hline$\Sigma\left[\mathrm{O}_{\mathrm{II}}\right] \lambda 7325$ & 23.8 & 5.09 & - & 4.30 \\
\hline [S III] $\lambda 9072$ & 28.2 & 27.7 & - & - \\
\hline [S III] $\lambda 9535$ & 67.7 & 68.8 & - & - \\
\hline$c_{\mathrm{ext}}$ & 0.45 & - & 0.45 & - \\
\hline$T_{\mathrm{e}}([\mathrm{O} \mathrm{III}])[\mathrm{K}]$ & 18300 & 18300 & 18300 & 18300 \\
\hline$n_{\mathrm{e}}\left[\mathrm{cm}^{-3}\right]$ & $\leq 10$ & $\leq 10$ & $\leq 10$ & $\leq 10$ \\
\hline$v_{\text {hel }}\left[\mathrm{km} \mathrm{s}^{-1}\right]$ & $161 \pm 23$ & - & $157 \pm 27$ & - \\
\hline
\end{tabular}

Table 3. Derived abundances (with representative errors for slit $s 1$ ) of the candidate WR nebula compared to averaged abundances of both EHRs and the central H II-region in NGC 55 (Tüllmann et al. 2003).

\begin{tabular}{lrrrr}
\hline \hline Parameter & slit $s 1$ & slit $s 2$ & EHRs & HR \\
\hline $12+\log (\mathrm{He} / \mathrm{H})$ & $11.36 \pm 0.05$ & 11.16 & 10.90 & 10.94 \\
$12+\log (\mathrm{O} / \mathrm{H})$ & $7.84 \pm 0.08$ & 7.83 & 7.72 & 8.05 \\
$\log (\mathrm{N} / \mathrm{O})$ & $-1.35 \pm 0.05$ & -1.44 & -1.45 & -1.26 \\
$\log (\mathrm{Ne} / \mathrm{O})$ & $-0.89 \pm 0.15$ & -0.49 & - & -0.85 \\
$\log (\mathrm{S} / \mathrm{O})$ & $-1.40 \pm 0.10$ & -1.38 & -1.80 & -1.41 \\
$\log \left(\mathrm{O}^{+} / \mathrm{O}\right)$ & -0.671 & -0.469 & -0.130 & -0.731 \\
$\log \left(\mathrm{S}^{+} / \mathrm{S}^{++}\right)$ & -0.503 & - & - & -0.722 \\
$\overline{Z / Z_{\odot}}$ & 0.13 & 0.13 & 0.11 & 0.23 \\
\hline
\end{tabular}

\subsection{The nature of the nebula}

The detection of nebular He II $\lambda 4686$ emission in both spectra puts some constraints on the ionizing source of the nebula. Since $54.4 \mathrm{eV}$ are required to ionize helium completely, only the hottest $\mathrm{O}$ stars or their evolutionary products (WR stars) provide sufficient luminosities at these photon energies. However, non-thermal ionizing mechanisms, such as in supernova remnants (SNRs), are not a priori excluded at this point.
Another constraint which also implies a hot atmosphere, if photoionization is chosen, comes from the faintness of any probable stellar continuum in the "red" wavelength region, which seems to become stronger towards the "blue", and apparently is strongest on UV broadband data collected with the optical monitor (OM) of the XMM-Newton satellite.

Further support of an extreme ionizing source is given by the high gas temperature of about $18300 \mathrm{~K}$ at a metal abundance which is otherwise comparable to the range of abundances observed for other nebulae in NGC 55. These H IIregions have typical gas temperatures of only $11500 \mathrm{~K}$ and oxygen abundances of about $10 \% Z_{\odot}$ (Tüllmann et al. 2003).

XMM-Newton EPIC-pn data obtained at different energy bands and overlaid onto the VLT $\mathrm{H} \alpha$-image (Fig. 5) reveal a significant detection of hard and soft X-rays around the northern part of the nebula. This emission is too well aligned with the outer optical border of the nebula to be just a normal lineof-sight effect. The questions if the lack of X-ray photons to the south of the nebula is caused by absorption of the ambient ISM (see Fig. 1) and if the extended X-ray emission in the north is related to the star formation activity in the disk below, remains yet to be answered.

In principle shock ionization could also be a possible ionization mechanism of the gas in the nebula under discussion. However, the observed emission line characteristics clearly indicate that the nebula is likely photoionized rather than shock ionized: in particular the weakness of [S II], [S III], and [N II] emission lines; the absence of Mg II emission and the weakness of [OI] $\lambda 6300$. Diagnostic diagrams such as those from Baldwin et al. (1981) or Veilleux \& Osterbrock (1987), and a comparison with shock models (Shull \& McKee 1979; Raymond 1979; Dopita \& Sutherland 1995) also indicate photoionization conditions.

In order to check the hypothesis that the dominant ionizing sources are massive stars, observed emission line strengths are compared to predictions made by NAT in an iterative process. The best match between model and observations was achieved using a model atmosphere for a WR star. In this case a pure-helium extended atmosphere of $T=90000 \mathrm{~K}$ (Wessolowski et al. 1988) was chosen. Results are shown in Table 2 in the columns labeled "NAT". They are in very good agreement with the observations and support the idea that TR 001507.7-391206 is photoionized by evolved massive stars.

Since NAT cannot predict $\mathrm{He}$ II/H line ratios, we now estimate the theoretical $\mathrm{He} \mathrm{II} / \mathrm{H} \beta$ line ratio and comparing it to the observed one (cf. Table 2). Following Osterbrock (1989), this ratio can be written as:

$\frac{\mathrm{He} \text { II } \lambda 4686}{\mathrm{H} \beta} \approx \frac{\int N_{\mathrm{He}^{++}} N_{\mathrm{e}} \alpha_{\lambda 4686}^{\mathrm{eff}}\left(\mathrm{He}^{+}, T\right) \mathrm{d} V_{\mathrm{He}^{++}}}{\int N_{\mathrm{p}} N_{\mathrm{e}} \alpha_{\mathrm{H} \beta}^{\mathrm{eff}}\left(H^{0}, T\right) \mathrm{d} V_{\mathrm{H}^{+}}}$,

where $N_{\mathrm{He}^{++}}, N_{\mathrm{p}}$, and $N_{\mathrm{e}}$ represent ion, proton, and electron densities, respectively. $\alpha^{\text {eff }}$ denotes the effective recombination coefficient of the corresponding transition whereas $\mathrm{d} V$ addresses the respective volume of the $\mathrm{H}^{+}$and $\mathrm{He}^{++}$-zone covered by the slit.

We assume that $\mathrm{H}$ is completely ionized and that $r_{\mathrm{He}^{++}}$, the radius of the $\mathrm{He}^{++}$-zone, is about $0.2 r_{\mathrm{H}^{+}}$. This is reasonable for 


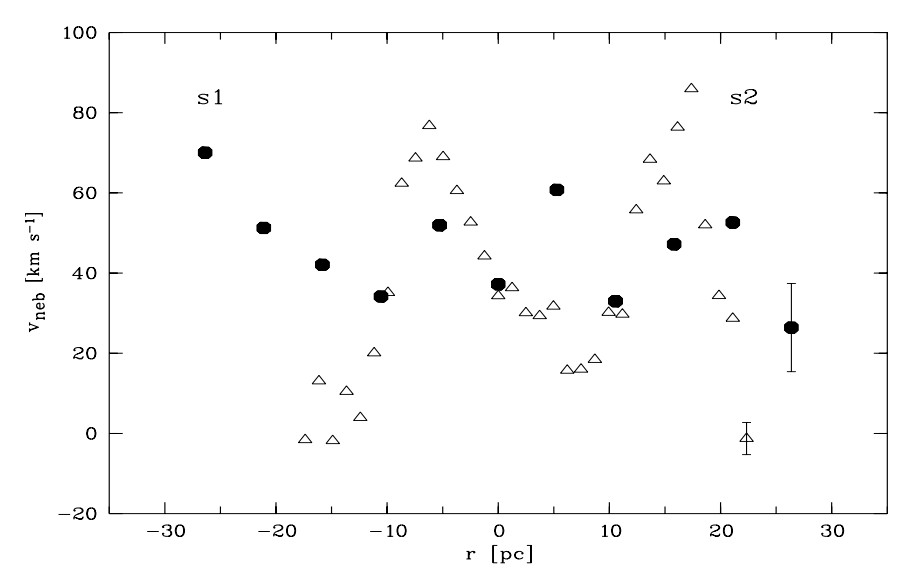

Fig. 6. Nebular $\mathrm{H} \alpha$-velocities as a function of radius $r$ plotted for both slit positions. A correction for the systemic velocity has been applied, assuming $v_{\mathrm{sys}}=118 \mathrm{~km} \mathrm{~s}^{-1}$ (Puche et al. 1991). Typical uncertainties in velocity are in the range of $4-12 \mathrm{~km} \mathrm{~s}^{-1}$ (see errorbars). Negative radial coordinates for $s 1(s 2)$ represent the western (southern) part of the nebula.

nebulae ionized by stars with effective temperatures of the order of $10^{5} \mathrm{~K}$, e.g., planetary nebulae. Then $V_{\mathrm{He}^{++}} / V_{\mathrm{H}^{+}}$amounts to 0.04 . Using tabulated recombination coefficients for He II and $\mathrm{H} \beta$ (Osterbrock 1989, Tables 4.2 and 4.3) and the approximation that for high ionization nebulae $N_{\mathrm{He}^{++}} / N_{\mathrm{H}^{+}} \approx N_{\mathrm{He}} / N_{\mathrm{H}} \approx$ 0.1 , we finally derive: $\mathrm{He} \operatorname{II} \lambda 4686 / \mathrm{H} \beta \approx 0.044$. As this is in good agreement with observational data, it appears to be justified to consider very hot massive stars, such as O3 or WR stars, to be the ionizing sources of TR 001507.7-391206.

We now derive an estimate of the number of such ionizing stars by converting the integrated $\mathrm{H} \alpha$-flux into a luminosity, assuming a distance to NGC 55 of $D=1.6 \mathrm{Mpc}$, and comparing this value to what is expected from theory. For slit $s 1$ the integrated $\mathrm{H} \alpha$-flux amounts to $F_{\mathrm{H} \alpha, \mathrm{s} 1}=1.54 \times 10^{-13} \mathrm{erg} \mathrm{s}^{-1} \mathrm{~cm}^{-2}$. At slit position $s 2$ we measure a value for $F_{\mathrm{H} \alpha, \mathrm{s} 2}$ of $2.26 \times$ $10^{-13} \mathrm{erg} \mathrm{s}^{-1} \mathrm{~cm}^{-2}$. This translates into the following luminosities: $L_{\mathrm{H} \alpha, \mathrm{s} 1}=4.7 \times 10^{37} \mathrm{erg} \mathrm{s}^{-1}$ and $L_{\mathrm{H} \alpha, \mathrm{s} 2}=6.9 \times 10^{37} \mathrm{erg} \mathrm{s}^{-1}$. Since the NAT-modeling of the observed emission line data yielded a typical effective temperature of $90000 \mathrm{~K}$ at a metallicity of $10 \%$ solar, such stars are expected to have a photon luminosity of the $\mathrm{H}$ I ionizing continuum of about $1.58 \times 10^{49} \mathrm{~s}^{-1}$ (Smith et al. 2002). Recombination line luminosities are calculated using case $\mathrm{B}$ recombination coefficients for $\mathrm{H}$ as given in Table 2.1 of Osterbrock (1989). From this data we derive expected stellar photon luminosities of $L_{\mathrm{H} \alpha}=5.6 \times 10^{37} \mathrm{erg} \mathrm{s}^{-1}$. If we scale this by 0.39 , the fraction of the nebular volume which each slit covers, we finally derive a corrected luminosity of $L_{\mathrm{H} \alpha}=2.2 \times 10^{37} \mathrm{erg} \mathrm{s}^{-1}$. This would be consistent with observed $\mathrm{H} \alpha$-luminosities if the central ionizing source consists of two to three WR stars.

It would be very interesting to obtain a much deeper spectrum of the central continuum sources in order to investigate the nature of these stars in detail. The presence of WR-typical broad emission lines as well as absorption features at wavelengths where helium and hydrogen lines do not coincide (e.g., at $4200 \AA$ or $4542 \AA$ ), would be indicators for the mix of early $\mathrm{O}$ and WR stars.

\subsection{Nebular kinematics}

Measurements of radial velocities determined from slit $s 2$, $10^{\prime \prime}$ south of TR 001507.7-391206 (dust cloud), reveal values ranging from $-1 \mathrm{~km} \mathrm{~s}^{-1}$ to $+45 \mathrm{~km} \mathrm{~s}^{-1}$. As this range is identical to velocities found for the nebula (Fig. 6), it implies that this object is indeed located on top of a huge gas spike.

Data for $s 2$ show that the ionized gas at the outskirts of the nebula meets the systemic velocity (Fig. 6) and is therefore mostly at rest. Hence these regions might be coincident with the outer (stationary) border of the nebula. At $s 1$ the kinematics looks completely different and the systemic velocity is never approached. This tells at least that TR 001507.7-391206 is not expanding symmetrically.

\subsection{Comparison with other objects}

Although direct observational evidence of the exact stellar types is lacking in the case of TR 001507.7-391206, the overabundance of $\mathrm{He}, \mathrm{S}$, and possibly $\mathrm{Ne}$ as well as the observed range of velocities seem to imply that we are faced with a massloss bubble very much like those around galactic WR stars. Since the stellar ionizing sources for TR 001507.7-391206 are apparently very hot, a suitable candidate for comparison would be the galactic nebula G2.4+1.4. This extraordinary mass-loss bubble around WR 102, a WO-type WR star, has been described in detail by e.g. Treffers \& Chu (1982), Dopita et al. (1990), Dopita \& Lozinskaia (1990), or Esteban et al. (1993). WR 102 is presumably the hottest and most evolved massive WR star known in the Galaxy. A comparison between $\mathrm{H} \alpha$ images of G2.4+1.4 and TR 001507.7-391206 reveals remarkable morphological similarities if this object would be projected to the distance of NGC 55.

If the equivalences between the WR nebulae in NGC 55 and the Galaxy hold true then TR 001507.7-391206 harbors one of the most massive and short-lived stars of NGC 55. Clearly, deep exposures using the VLT would be required to obtain spectra of the central sources with sufficient signal to noise.

\section{Summary}

We have collected multi-wavelength data in order to constrain the nature of a high ionization, high temperature nebula located in the diffuse ISM near the main body of NGC 55. Its emission line characteristics, gas temperature, and elevated $[\mathrm{He} / \mathrm{H}],[\mathrm{S} / \mathrm{O}]$, and possibly $[\mathrm{Ne} / \mathrm{O}]$-abundance are consistent with slightly enriched, plowed matter present either in SNRs or WR nebulae and ionized by a hot continuum. Optical and UV broadband data reveal the existence of continuum emission originating from this object which directly excludes SNRs. A SNR nature is also made improbable, because the characteristic emission of Mg II and [O I] 66300 is absent. Moreover, observed emission line intensities compare well with photoionization models using a hot WR atmosphere of $T=90000 \mathrm{~K}$ as input.

The most plausible interpretation of the data is a WR nebula photoionized by one of the hottest and most massive WR stars (WO) similar to the nebula G2.4+1.4 in the Galaxy which is 
ionized by WR 102. Such stars are rather inconspicuous in optical imaging, but very deep longslit spectra with the VLT across the nebula might well reveal the characteristic broad emission lines of the central WR object.

Acknowledgements. R.T. received financial support by the SFB 591 and DLR through grant 50OR0102. The referee C. Leitherer pointed out some aspects which made the paper more conclusive.

\section{References}

Baldwin, J. A., Phillips, M. M., \& Terlevich, R. 1981, PASP, 93, 5

Christensen-Dalsgaard, J. 1998, Space Sci. Rev., 85, 19

De Robertis, M. M., Dufour, R. J., \& Hunt, R. W. 1987, JRASC, 81, 195

Dopita, M. A., Lozinskaya, T. A., McGregor, P. J., \& Rawlings, S. J. 1990, ApJ, 351, 563

Dopita, M. A., \& Lozinskaya, T. A. 1990, ApJ, 359, 419

Dopita, M. A., \& Sutherland, R. S. 1995, ApJ, 455, 468

Esteban, C., Smith, L. J., Vilchez, J. M., \& Clegg, R. E. S. 1993, A\&A, 272,299
Ferguson, A. M. N., Wyse, R. F. G., \& Gallagher, J. S. 1996, AJ, 112, 2567

Graham, J. A., \& Lawrie, D. G. 1982, ApJ, 253, L73

Grevesse, N., \& Sauval, A. J. 1998, Space Sci. Rev., 85, 161

Mathis, J. S., \& Rosa, M. R. 1991, A\&A, 245, 625

Mason, K. O., Breeveld, A., Much, R., et al. 2001, A\&A, 365, L36

Osterbrock, D. E. 1989, Astrophysics of Gaseous Nebulae and Active Galactic Nuclei (University Science Books)

Otte, B., \& Dettmar, R.-J. 1999, A\&A, 343, 705

Puche, D., Carignan, C., \& Wainscoat, R. J. 1991, AJ, 101, 447

Raymond, J. C. 1979, ApJS, 39, 1

Shull, J. M., \& McKee, C. F. 1979, ApJ, 227, 131

Smith, L. J., Norris, R. P. F., \& Crowther, P. A. 2002, MNRAS, 337, 1309

Strüder, L., Briel, U., Dennerl, K., et al. 2001, A\&A, 365, L18

Treffers, R. R., \& Chu, Y.-H. 1982, ApJ, 254, 132

Tüllmann, R., Rosa, M. R., Elwert, T., et al. 2003, A\&A, 412, 69

Veilleux, S., \& Osterbrock, D. E. 1987, ApJS, 63, 295

Wessolowski, U., Schmutz, W., \& Hamann, W.-R. 1988, A\&A, 194, 160 